\title{
Three-Dimensional Visualization of Powder Motion in a High Shear Mixer
}

\author{
Yoshitoshi Saito $^{1, * 1}$, Andy Ingram ${ }^{2, * 2}$, Xianfeng Fan $^{2, * 3}$ and Jonathan Peter Kyle Seville ${ }^{1, * 4}$ \\ ${ }^{1}$ Department of Chemical Engineering, University of Birmingham, Birmingham B15 2TT, UK \\ ${ }^{2}$ Positron Imaging Centre, School of Physics and Astronomy, University of Birmingham, Birmingham B15 2TT, UK
}

This study provides new findings on the effects of blade speed (300 versus $450 \mathrm{rpm}$ ) using a three-blade flat impeller during dry powder granulation within a high shear mixer. It was possible to achieve roping behavior, which is recommended for achieving good liquid distribution and nucleation, with flat blades at two different impeller speeds. [doi:10.2320/matertrans.M2010428]

(Received December 20, 2010; Accepted May 18, 2011; Published July 25, 2011)

Keywords: positron emission particle tracking, high shear mixer, particulate motion, powder mixing

\section{Introduction}

A particle granular flow is a two-phase flow of collections of discrete solid particles dispersed in an interstitial fluid, where the gaseous phase plays a negligible role in the flow mechanics. The dominant mechanism affecting the flow behavior of the granular material is the random motions of particles resulting from the interactive collisions between them. Because these random motions of particles in a granular flow are analogous to the motion of molecules in a gas, the dense-gas kinetic theory ${ }^{1)}$ is applied to analyze and model the granular flow behavior.

Mixing of granular materials has economic importance in the manufacture of various products, such as foods, pharmaceuticals, detergents, chemicals, and plastics. In many cases, a better mixing process would greatly increase the quality and value of products, and mixing is therefore regarded as a key process. The subject of granular mixing in several types of mixers has attracted the interest of a number of researchers in recent years. Experimental tests and computer simulations have been performed for convective and dispersive mixing mechanisms that occur in a rotating tumbler, ${ }^{2)}$ in a Vblender, ${ }^{3)}$ and in a tote blender. ${ }^{4)}$ Computer simulation has also become a powerful tool for studying mixing behaviors in a granular flow system.

Powder mixing is a fundamental technology used in a variety of industries. The quality of the final product is affected by several important factors, including the degree of mixing of the constituent materials, the properties of the source materials which can affect flow patterns, and the nature of the mixing procedure which will influence the homogeneity of the resulting mixture. In order to enhance process understanding process and to develop predictive process models in high shear granulation, there is an ongoing search for simulation tools and experimental methods to

\footnotetext{
${ }^{* 1}$ Present address: Environment \& Process Technology Center, Nippon Steel Corporation, Chiba 293-8511, Japan. Corresponding author, E-mail: saito.yoshitoshi@nsc.co.jp

*2Present address: Department of Chemical Engineering, University of Birmingham, Birmingham B15 2TT, UK

${ }^{* 3}$ Present address: Institute for Materials and Processes, School of Engineering, The University of Edinburgh, Edinburgh, EH9 3JL, UK

${ }^{* 4}$ Present address: School of Engineering, University of Warwick, Coventry, CV4 7AL, UK
}

model and measure the velocity and shear fields in the mixer. An understanding of the mixing of powders is vital because of its major impact on the performance of many unit operations and the behavior of many products. However, little is known about the behavior of particles within process equipment. Flow visualization is essential to understanding the phenomena of powder mixing, and process tomography has been undertaken for the validation of computational fluid dynamic models and for the possibility of constructing a flowmeter for multi-phase flow. Positron Emission Particle Tracking (PEPT) is widely used in science and engineering to obtain detailed information on the motion and flow fields of fluids or granular materials in multiphase systems. ${ }^{5)}$ PEPT can provide quantitative information regarding particle motion in a mixer, and it has been used to study particle flow in different regions of a high shear mixer. ${ }^{6,7)}$

In this study, the motion of particles in a vertical cylindrical mixer with three flat blades was studied throughout the bed using PEPT, which revealed the location of tracer particles many times per second.

\section{Experimental Method}

\subsection{The PEPT system}

Positron Emission Particle Tracking (PEPT) was invented in the 1980's at the University of Birmingham as a refinement of Positron Emission Tomography (PET). PEPT is a state-ofthe-art technique for particle research, being one of the few available non-invasive methods to visualize and quantify the particle motion. PEPT has been applied to dynamic processes found in various engineering applications, where it characterizes the particle motion in operations. Recently, the authors have shown that PEPT also offers great possibilities to develop our understanding of a granulation process in high shear mixer. ${ }^{6,7)}$

The basic principle of PEPT is based on positron annihilation. Using PEPT, one single radioactive tracer particle is introduced in the system of interest. Upon decay, this tracer will emit positrons which subsequently annihilate with a neighboring electron. As shown in Fig. 1 each annihilation results in a pair of back-to-back $511 \mathrm{keV} \gamma$-rays that are detected in coincidence by two parallel detectors. A line along which the annihilation has occurred is defined. Using a number of successive detected lines enables the tracer to be 


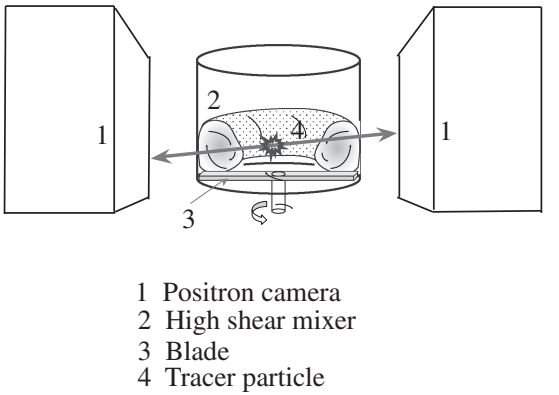

Fig. 1 Experimental apparatus.

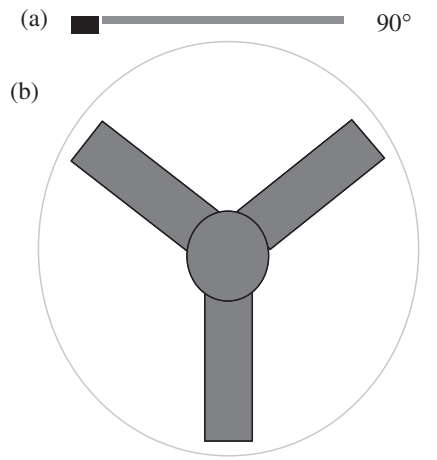

Fig. 2 Blade design and configuration. (a) Side view and (b) top view of 3 flat blades.

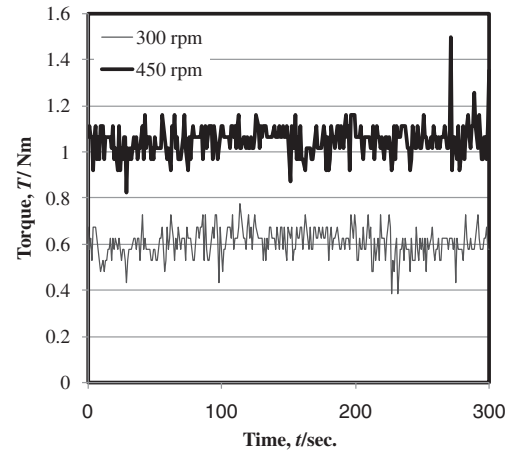

Fig. 3 Torque measurements using three flat blades. located in three dimensions by triangulation. As some detected events may be corrupted due to scattering of the $\gamma$ rays, an iterative algorithm is used to optimize the tracer location assessment. PEPT tracks a small radioactively labeled particle at speeds up to $10 \mathrm{~m} / \mathrm{s}$. Simultaneous detection of the two gamma rays in an array of detectors (The Birmingham PEPT camera) defines a line along which the annihilation occurred. In PEPT measurements, the tracer is representative of the bulk or of an intended fraction of the bulk, and therefore its average motion is obtained. The tracer activity needs to be high enough to cover relevant operating timescales. The experiment duration is long enough to allow the tracer to visit all possible locations in the experimental setup several times. Among the available radioisotopes, ${ }^{18} \mathrm{~F}$ (halflife $109 \mathrm{~min}$ ) is often used as finer tracer. After cyclotron bombardment using a $33 \mathrm{MeV} 3 \mathrm{He}$ beam, ${ }^{18} \mathrm{~F}$ in a freely ionic state was produced from purified water and could be transferred to the selected tracer particle via surface adsorption.

\subsection{PEPT applied to high shear mixer}

Microcrystalline cellulose powder (MCC, Avicel PH102, Merck, UK) was used as the starting material. The high shear mixer used in this study was fitted with a stainless steel bowl (internal diameter $210 \mathrm{~mm}$, vertical side height $200 \mathrm{~mm}$ ) attached by using a centrally mounted impeller rotating around the vertical axis. Figure 1 shows the experimental apparatus used to dry mix the materials. The blade geometry is shown in Fig. 2; a three-blade flat impeller with an angle of $90^{\circ}$ was used.

A resin particle (a strong base anion ion exchanger) with a diameter of $200 \mu \mathrm{m}$ and a density of $1100 \mathrm{~kg} \cdot \mathrm{m}^{-3}$ was used as the radiolabeled tracer particle in PEPT in order to match the diameter and density of the MCC powder. The dry powder was blended in the high shear mixer for $300 \mathrm{~s}$. To examine the effects of blade speed on the powder mixing, the rotational speed was varied (350 and $400 \mathrm{rpm}$ ). The torque that was generated in the high shear mixer as a result of the friction at the powder/wall interface was measured in order to control the granulation process and the endpoint. The bowl was mounted on a ball-race so that it was free to rotate about the axis, but was constrained from doing so by a torque arm, which presses against a calibrated strain gauge. ${ }^{8)}$ This provides a direct measure of torque on the bowl.

The experiment procedure involved placing a mass of powder into the bowl. The mixer was used as a rheometer; that is, as the mixer bowl rotated, the stainless steel plate attached to the mixer bowl generated impact forces on the strain gauge, based on which torque data was obtained at 1-s intervals. The bowl was mounted on bearings, which supported the bowl and allowed it to rotate freely in a horizontal plane. Rotation was prevented by a load arm that was fixed to the bowl and which rested against a load cell mounted on the frame of the apparatus. The torque was computed from the measured load. The drive shaft, which was mounted on bearings, projected into the center of the bowl and allowed an impeller blade to be attached. The impeller blade was manufactured to fit the bowl with a tip clearance in the range of 0.5 to $1 \mathrm{~mm}$. The shaft was driven by a $7.5-\mathrm{kW}$ three-phase DC-drive electric motor, the speed of which could be altered continuously in the range of 300$600 \mathrm{rpm}$. The speed could be controlled to within $\pm 5 \mathrm{rpm}$. Signals for the torque and the speed were sampled at 0.5$1 \mathrm{~Hz}$ by a data logger.

\section{Results and Discussion}

Noting that torque is not only important as a means of monitoring and controlling the agglomeration processes but also as a parameter to understand mixer design based on its sensitivity to operating conditions, Knight et al. ${ }^{9)}$ studied the factors that could affect the impeller torque of a vertical high shear mixer, using sand as the experimental material and using both disc and blade impeller designs. They reported that torque was independent of the disc speed when using a disc impeller, whereas the use of blade impellers resulted in torque that varied considerably with the height and bevel angle. Figure 3 shows the torque data obtained in the present study for the flat blade design: torque increased significantly at the higher rotational speed of $450 \mathrm{rpm}$.

Knight et al. ${ }^{9)}$ also found that torque varied considerably with both the height and bevel angle of the blades in the vertical high shear mixer. In their study, the torque value of flat blades increased linearly with an increase of blade speed at speeds above $100 \mathrm{rpm}$. When using beveled blades, however, torque increased slightly with increasing rotational speed, and decreased again at high speeds. They reported that both frictional and inertial interactions of the powder with the blades accounted for this difference. It has been reported by Knight et al. ${ }^{9)}$ that the frictional force made a significant contribution to the total torque when using low-angle beveled blades. 
As mentioned above, the effect of the rotational speed of flat blades on the torque in this study-where torque increased significantly at the higher rotational speedcorresponds to the results for flat blades obtained by Knight et al. ${ }^{9)}$ However, Knight et al. did not show the powder flow patterns or velocity distributions in their paper. As Knight ${ }^{10)}$ suggests that both blade shape and rotational speed are important variables in a high shear mixer, the dry powder motion was observed visually using PEPT in the present study in order to investigate the effects of rotational speed and blade type on the flow of the material. The data for both the flow patterns and velocity distributions can be shown in a side view of the mixer, given by displaying the time-averaged occupancy information as a function of position.

The motion of the particle can be replayed in three orthogonal vies as shown in Fig. 4. The PEPT trajectory in a $\mathrm{XY}$-, YZ- and XZ orthogonal projection is shown together with a graph of the tracer velocity as a function of time. A fourth window shows the instantaneous speed of the tracer. Based on these results, the data are presented as a combined occupancy and velocity vector plot. in Fig. 5. The results displayed are averaged over all the passes of the tracer through each individual pixel. The length of the arrows corresponds to velocity of the flow. The quantity in the occupancy plot represents the fraction of the selected time range that the tracer was located in each pixel. Occupancy $O$ is defined as $O=$ $t_{\text {pixel }} / t_{\text {total }}$, in which $t_{\text {pixel }}$ is the time the trace spends within an individual pixel, and $t_{\text {total }}$ is the total time range. As is clear from the pink colored regions in Fig. 5, there was roping behavior at both 300 and $450 \mathrm{rpm}$ with flat blades, indicating good bed turnover and stable flow, and the bed height increased slightly with an increase in rotational speed. In "Roping behavior", material from the bottom of the powder bed was pushed out and up the vessel wall and then tumbled down the angled bed surface towards the centre of the bowl. There was both good rotation of the bed and good vertical turnover. ${ }^{11)}$ The rotational speed had a significant effect on the average speed of particles near the blades, but had little effect on the average speed of particles at the surface of the power bed.

Velocity was calculated using the six point method for PEPT. ${ }^{12)}$ The six point method is the most commonly used method due to the fact that it can reduce the effect of error in the PEPT measurements. $V_{i}$ is the velocity at the $i$ th particle track location, which is point $p_{i}$ in space and at time $t_{i}$. The velocity $v_{i}$ at point $p_{i}$ and time $t_{i}$ can be calculated from six data points using the following formula.

$$
\begin{aligned}
v_{i}= & 0.1\left(\frac{p_{i+5}-p_{i}}{t_{i+5}-t_{i}}\right)+0.15\left(\frac{p_{i+4}-p_{i-1}}{t_{i+4}-t_{i-1}}\right) \\
& +0.25\left(\frac{p_{i+3}-p_{i-2}}{t_{i+3}-t_{i-2}}\right)+0.25\left(\frac{p_{i+2}-p_{i-3}}{t_{i+2}-t_{i-3}}\right) \\
& +0.15\left(\frac{p_{i+1}-p_{i-4}}{t_{i+1}-t_{i-4}}\right)+0.1\left(\frac{p_{i}-p_{i-5}}{t_{i}-t_{i-5}}\right)
\end{aligned}
$$

The velocity fields calculated using this method are accurate to approximately $10 \% .{ }^{11)}$ Figure 6 shows the velocity histograms at the rotational speed of $300 \mathrm{rpm}$. These velocity histograms were constructed for the visualization and initial analysis of the transferred PEPT data using the "Track" program for Windows (this software was produced
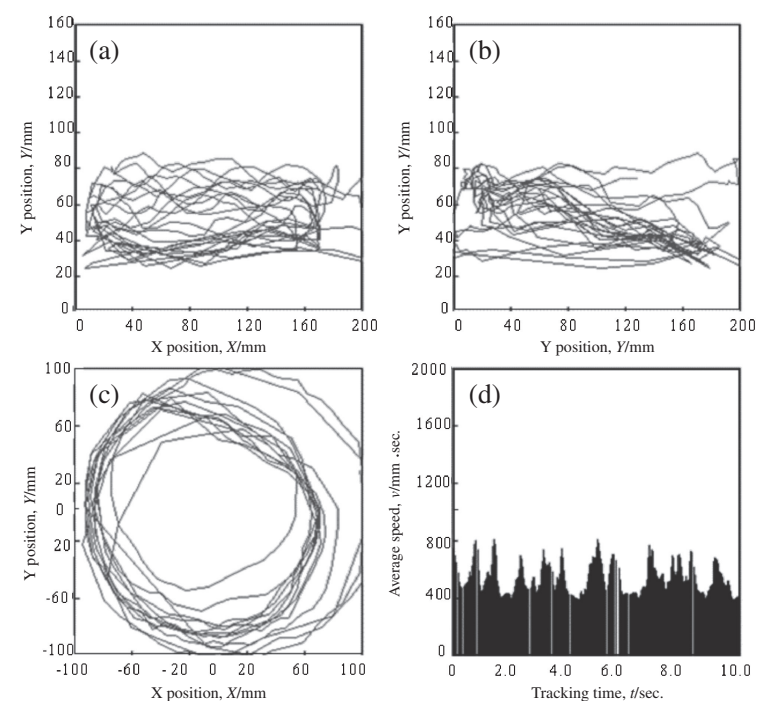

Fig. 4 Tracer locations and average speed obtained from the PEPT trajectory with a time (a) 0-10s (b) 0-100 s (c) 100-200 s (d) 200-300 s.

by Dr. David Parker at Birmingham University but has not been published). The histograms of the velocity distribution and the residence time distribution within a specified region were obtained using "Track" because the "Track" analysis covers the history of the particle's location over time. For example, at an axial position of $30-40 \mathrm{~mm}$ for the rotational speed of $300 \mathrm{rpm}$, the mean axial velocity and mean radial velocity were $47.95 \mathrm{~mm} / \mathrm{s}$ and $193.91 \mathrm{~mm} / \mathrm{s}$, respectively, with a mean particulate speed of $1088.78 \mathrm{~mm} / \mathrm{s}$.

Figure 7 shows the averaged velocity distribution for the two rotational speeds as a function of axial position and mean velocity. The position of the blade in the Z-direction was between 20 and $30 \mathrm{~mm}$. In Fig. 7, the powder and granule bed are divided layer by layer at every $10 \mathrm{~mm}$ of depth in the axial direction in order to obtain the average velocities along the bed height. For instance, the average velocity at the axial position of $30 \mathrm{~mm}$ corresponded to the velocity averaged between 25 and $35 \mathrm{~mm}$. The average velocity distribution as well as the flow pattern during granulation, in which the powder and granule bed were divided layer by layer in order to obtain the average velocity along the bed height, is shown in Fig. 6. This velocity distribution was calculated using the six point method described above. Further, the distribution of the velocities at $450 \mathrm{rpm}$ particularly near the blades was greater than at $300 \mathrm{rpm}$. This indicates that there are marked differences both in the swept volume and in the flow pattern between the two rotational speeds. The swept volume at $450 \mathrm{rpm}$ was much larger than that at $300 \mathrm{rpm}$, particularly near the blades. The difference of the particle velocity between $300 \mathrm{rpm}$ and $450 \mathrm{rpm}$ became smaller as the axial position approached the surface of the powder bed. The velocity in the surface region of the powder bed at $450 \mathrm{rpm}$, however, was almost the same as that at $300 \mathrm{rpm}$. As is clear from the figure, the flat blade design resulted in a velocity that became constant in areas away from the blade. At an axial position of 80 to $105 \mathrm{~mm}$, which is near the surface, the velocity was approximately $500 \mathrm{~mm} \mathrm{~s}^{-1}\left(0.5 \mathrm{~m} \mathrm{~s}^{-1}\right)$. Moreover, the velocity at this position showed a tendency to be independent of both the rotational speed. This surface velocity 
(a)

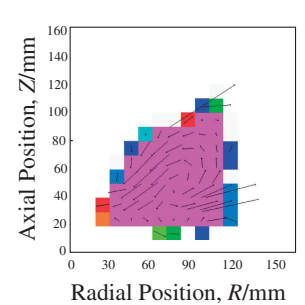

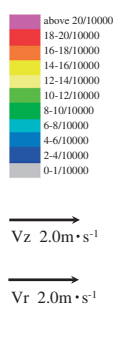

(b)

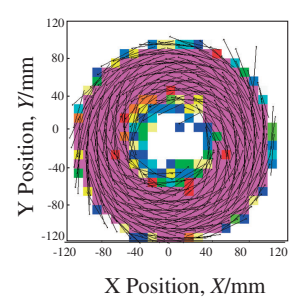

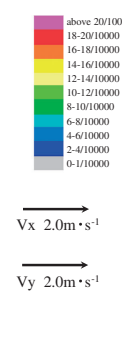
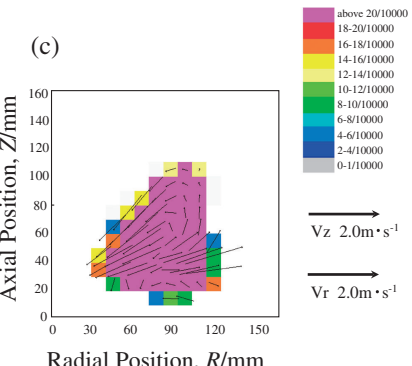

(d)

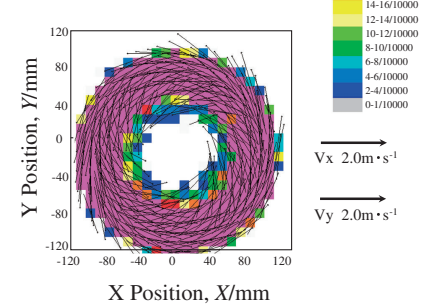

Fig. 5 Combined occupancy and velocity vector plots at $300 \mathrm{rpm}$ and $450 \mathrm{rpm}$ when using three flat blades. (a) $300 \mathrm{rpm}$ (side view) (b) $300 \mathrm{rpm}$ (top view) (c) $450 \mathrm{rpm}$ (side view) (d) $450 \mathrm{rpm}$ (top view).

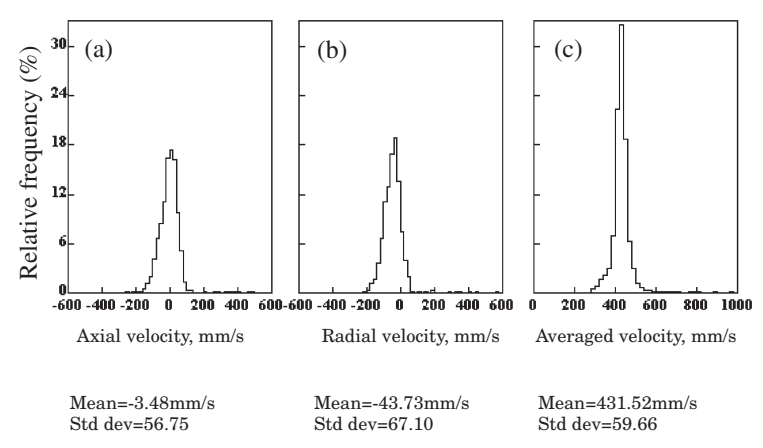

Fig. 6 Velocity histograms from PEPT measurement for 3 flat blades, 300 rpm. (a) Axial velocity, (b) radial velocity, (c) overall speed.

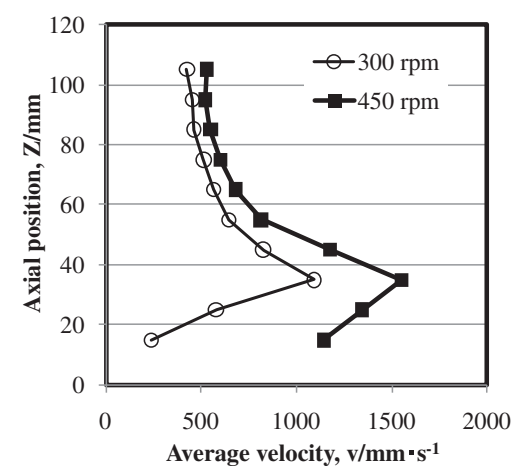

Fig. 7 Average velocity distribution.

also corresponds approximately to that in the previous study ${ }^{7)}$ where the three beveled blade were used. It is very interesting that particle velocity near the blades increased with rotational speed of the flat blades, but the effect of rotational speed on particle velocity became smaller with axial position.

\section{Conclusion}

This study investigated the mixing behavior of granular materials in a vertical high shear mixer. Image processing technology and a particle tracking method measured the effect of blade speed ( 300 versus $450 \mathrm{rpm}$ ) during dry powder mixing.

This study provides new findings in relation to flat blades: the particle velocity near the blade increased with rotational speed of blade, but the effect of rotational speed on particle velocity became smaller with axial position. The velocity in the surface region of the powder bed at $450 \mathrm{rpm}$ was almost the same as at $300 \mathrm{rpm}$. It is very interesting that the surface velocity also corresponds approximately to that in the previous study where the three beveled blade were used. This indicates that the velocity at the surface of the powder bed in high shear mixer shows a tendency to be independent of both the rotational speed and blade shape. It was possible to achieve roping behavior at both 300 and $450 \mathrm{rpm}$ with flat blades, indicating good bed turnover and stable flow, and the bed height increased slightly with an increase in rotational speed.

\section{Acknowledgement}

The present study was supported in part by Nippon Steel Corporation.

\section{REFERENCES}

1) S. B. Savage and D. J. Jeffrey: J. Fluid Mech. 110 (1981) 255-272.

2) J. J. McCarthy, D. V. Khakhar and J. M. Ottino: Powder Technology 109 (2000) 72-82.

3) M. Moakher, T. Shinbrot and F. J. Muzzio: Powder Technology 109 (2000) 58-71.

4) O. S. Sudah, D. Coffin-Beach and F. J. Muzzio: Powder Technology 126 (2002) 191-200

5) D. J. Parker and X. Fan: Particuology 6 (2008) 16-23.

6) Y. Saito, A. Ingram, X. Fan and J. P. K. Seville: Mater. Trans. 50 (2009) 2341-2343.

7) Y. Saito, X. Fan, A. Ingram and J. P. K. Seville: Chem. Eng. Sci. 66 (2011) 563-569.

8) M. Bardin: Granulation behaviour of silica in a high-shear mixer, (MPhil Thesis, University of Birmingham 2001).

9) P. C. Knight, J. P. K. Seville, A. B. Wellm and T. Instone: Chem. Eng. Sci. 56 (2001) 4457-4471.

10) P. C. Knight: Powder Technology 140 (2004) 156-162.

11) J. D. Litster, K. P. Hapgood, J. N. Michaels, A. Sims, M. Roberts and S. K. Kameneni: Powder Technology 124 (2002) 272-280.

12) R. L. Stewart, J. Bridgwater, Y. C. Zhou and A. B. Yu: Chem. Eng. Sci. 56 (2001) 4257-4271.

\section{Nomenclature}

$\mathrm{v}_{\mathrm{z}}$ : Velocity along $\mathrm{z}$-axis, $v / \mathrm{m} \cdot \mathrm{s}^{-1}$

$\mathrm{v}_{\mathrm{r}}$ : Velocity relative to the $\mathrm{Vz}$ axis, $v / \mathrm{m} \cdot \mathrm{s}^{-1}$

$p$ : particle track location, $p / \mathrm{m}$

$t$ : time of particle track location, $t / \mathrm{s}$

\section{Subscripts}

$i, j$ : corresponding to $i$ th, $j$ th particle track location $j$ is also used as a summation index, in difference equations $r$ : r-direction component $z$ : z-direction component 